\title{
Complex Dynamics in the Shen-Larter Model of Calcium Oscillations
}

\author{
Jianwei Hu ${ }^{a}$, Huiping Fang ${ }^{b}$ and Wei Fang ${ }^{1, c}$ \\ School of Mathematics and Statistics, Huangshan University, Xihai Road, Huangshan 245041, P. R. \\ China \\ aemail:jianweihu@hsu.edu.cn, bemail:fhp@hsu.edu.cn, cemail: sxxfw@hsu.edu.cn \\ ${ }^{1}$ Corresponding author
}

Keywords: Shen-Larter model, calcium oscillation, supercritical Hopf bifurcation, equilibrium, center manifold theory

\begin{abstract}
This paper discusses the nonlinear dynamics of Shen-Larter calcium oscillation model with the center manifold theorem and bifurcation theory. A numerical approach is provided to discuss the variation in classification and stability of equilibria with parameter value. It is shown that the critical values of parameter in this model are due to supercritical Hopf bifurcation of equilibria. This numerical simulation confirms the theoretical results of this research. Finally, a complete description of the complex dynamics of the Shen-Larter calcium oscillation model is provided by combining the numerical and theoretical results.
\end{abstract}

\section{Introduction}

Cytosolic calcium has shown its importance in the study of a large variety of cell types. Calcium has been linked to secretion, heart function, blood clotting, muscle contraction, and diseases of the muscular and nervous systems apart from cellular functions such as growth, gene expression, division, and apoptosis. Many activities of biological cells are controlled by calcium $\left(\mathrm{Ca}^{2+}\right)$ oscillations[1]. The mechanisms of delivering external signals to the inside of the cell are complicated, and calcium plays an important role in it. Commonly, by varying the concentration of $\mathrm{Ca}^{2+}$ inside the cell, signals take place, for example, by intracellular $\mathrm{Ca}^{2+}$ oscillations. Cells can control signals' processes in response to frequency changes. Hence, how $\mathrm{Ca}^{2+}$ oscillations effect the cell behaviors by controlling the signals is of vital scientific interest. Calcium oscillations are often organized into intracellular and intercellular calcium waves. Cells generate signals from transducer exogenous stimulation into physiological output. $\mathrm{Ca}^{2+}$ acts as an intracellular messenger in many cellular processes such as vesicle secretion and gene transcription[2].

Typically, $\mathrm{Ca}^{2+}$ oscillations arise by an agonist, such as a hormone or a neurotransmitter in response to external stimulation[3-9]. The agonist initiates a chain of reactions that leads to activation of phospholipase $C$ which then catalyses the formation of inositol $(1,4,5)$-trisphosphate (IP3). The agonist sticks to the receptors on the cell membrane. IP3 sticks to the receptors on the endoplasmic reticulum which is the cell's internal store of highly concentrated $\mathrm{Ca}^{2+}$ and diffuses through the cell cytoplasm. IP3 receptors are also $\mathrm{Ca}^{2+}$ signatures, and open when IP3 sticks, which leading to a large flux of $\mathrm{Ca}^{2+}$ from the endoplasmic reticulum into the cytoplasm. When IP3 are released from the receptors, the cell pumps $\mathrm{Ca}^{2+}$ back into the endoplasmic reticulum or $\mathrm{Ca}^{2+}$ them out of the cell.

Over the last decades, a large number of mathematical models are proposed to characterize changes of $\mathrm{Ca}^{2+}$ signals inside or outside cell membrane during transduction[10]. Shen and Larter[11] proposed a mathematical model which is described by some differential equations and some researchers made a deep study on it[10]. These researches concentrated on numerical simulations of the critical value of some parameters of calcium oscillations and rarely have notification on theoretical analysis. Chang and Chen[12] made a theoretical analysis of complex dynamics in Chen's system in 2006. They also performed some new numerical simulations which not only have confirmed the theoretical analysis results but also shown some new phenomena including homoclinic 
bifurcation, coexistence of two stable limit cycles, one chaotic attractor and some periodic solutions emerging from Hopf bifurcation.

In this paper, we further analyze Shen and Larter calcium oscillation model on the existence, types and stability of equilibria by qualitative theory and stability theory of dynamical models[13]. Also, we analyze the phenomenon of the appearance and disappearance of calcium oscillations with the center manifold theorem and bifurcation theory[14]. At last, we numerically simulate the theoretical analysis results to verify its rationality and accuracy.

\section{Mathematical Model}

The mathematical model proposed by Shen and Larter[4] is described by the following differential equations:

where

$$
\left\{\begin{array}{l}
d C a_{c y t} / d t=J_{c h}+J_{\text {leak }}-J_{\text {pump }}+J_{\text {in }}-J_{\text {out }} \\
d C a_{e r} / d t=J_{\text {pump }}-J_{c h}-J_{\text {leak }} \\
d I P_{3} / d t=J_{+}-J_{-}
\end{array}\right.
$$

$$
\begin{aligned}
& J_{c h}=k_{c h}\left(I P_{3}^{4} / I P_{3}^{4}+K_{1}^{4}\right)\left(K_{4} C a_{c y t} /\left(C a_{c y t}+K_{4}\right)\left(C a_{c y t}+K_{5}\right)\right)^{3} C a_{e r}, J_{\text {leak }}=k_{\text {leak }} C a_{e r}, J_{-}=k_{-} \cdot I P_{3}, \\
& J_{\text {pump }}=k_{\text {pump }} C a_{c y t}^{2} / C a_{c y t}^{2}+K_{2}^{2}, J_{\text {in }}=k_{\text {in } 1} \cdot r+k_{\text {in } 2}, J_{\text {out }}=k_{\text {out }} C a_{c y t}, J_{+}=k_{+} \cdot r \cdot C a_{c y t} / C a_{c y t}+K_{3} .
\end{aligned}
$$

Parameters for which all calculations are made if not otherwise stated: $k_{\text {ch }}=3000.0 \mu \mathrm{Ms}^{-1}, k_{\text {leak }}=1.0 \mathrm{~s}^{-1}, k_{\text {pump }}=50.0 \mu \mathrm{Ms}^{-1}, k_{\text {in } 1}=4.0 \mu \mathrm{Ms}^{-1}, k_{\text {in2 }}=1.0 \mu \mathrm{Ms}^{-1}$, $k_{\text {out }}=5.0 \mathrm{~s}^{-1}-30.0 \mathrm{~s}^{-1}, k_{+}=4.0 \mu \mathrm{Ms}^{-1}, k_{-}=2.0 \mu \mathrm{Ms}^{-1}, r=0.5, K_{1}=K_{2}=0.2 \mu M, K_{3}=1.0 \mu M$, $K_{4}=K_{5}=0.69 \mu \mathrm{M}$. In the following analysis and numerical simulations parameter $k_{\text {out }}$ is considered as bifurcation parameter since it is more easily amenable to experimental manipulation.

\section{Analysis of Stability and Bifurcation of Equilibria}

Let $x=C a_{c y t}, y=C a_{e r}, z=I P_{3}, r=k_{\text {out }}$, system (1) can be rewritten in the following form:

$$
\left\{\begin{array}{l}
d x / d t=985.527 x^{3} y z^{4} /\left((x+0.69)^{6}\left(z^{4}+0.0016\right)\right)+y-50 x^{2} /\left(x^{2}+0.04\right)+3-r x \\
d y / d t=50 x^{2} /\left(x^{2}+0.04\right)-y-985.527 x^{3} y z^{4} /(x+0.69)^{6}\left(z^{4}+0.0016\right) \\
d z / d t=2 x /(x+1)-2 z
\end{array}\right.
$$

According to the actual meaning of $\mathrm{x}, \mathrm{y}, \mathrm{z}$ and $\mathrm{r}$, only one condition needs to be studied whether there exists equilibria of system (2) when $r \in[5.0,30.0]$. Based on theoretical analysis we have:

When $r<7.49843$, there is a stable node of system (2); when $r=7.4984261$, system (2) has a non-hyperbolic equilibrium $O_{1}=(0.40008,1.2766,0.28576)$; when $7.49843<r<24.37676$, system (2) has an equilibrium (saddle); when $r=24.37676$, system (2) has a non-hyperbolic equilibrium $O_{1}=(0.12307,9.001,0.10958)$; when $r>24.37676$, there exists a stable node of system (2).

When $r=r_{0}$, the corresponding equilibrium of system (2) is $\left(x_{0}, y_{0}, z_{0}\right)$, and let $x_{1}=x-x_{0}$, $y_{1}=y-y_{0}, z_{1}=z-z_{0}, r_{1}=r-r_{0}$. In order to apply the center manifold theorem with parameters of a continuous dynamical system, $r_{1}$ is regarded as a new dynamical variable. Under the consideration of $d r_{1} / d t=0$ and system (2), we have: 


$$
\left\{\begin{aligned}
d x_{1} / d t= & 985.527\left(x_{1}+x_{0}\right)^{3}\left(y_{1}+y_{0}\right)\left(z_{1}+z_{0}\right)^{4} /\left(\left(x_{1}+x_{0}+0.69\right)^{6}\left(\left(z_{1}+z_{0}\right)^{4}+0.0016\right)\right) \\
& +\left(y_{1}+y_{0}\right)-50\left(x_{1}+x_{0}\right)^{2} /\left(\left(x_{1}+x_{0}\right)^{2}+0.04\right)+3-\left(r_{1}+r_{0}\right)\left(x_{1}+x_{0}\right) \\
d y_{1} / d t= & 50\left(x_{1}+x_{0}\right)^{2} /\left(\left(x_{1}+x_{0}\right)^{2}+0.04\right)-\left(y_{1}+y_{0}\right)-985.527\left(x_{1}+x_{0}\right)^{3} \\
& \left(y_{1}+y_{0}\right)\left(z_{1}+z_{0}\right)^{4} /\left(x_{1}+x_{0}+0.69\right)^{6}\left(\left(z_{1}+z_{0}\right)^{4}+0.0016\right) \\
d z_{1} / d t= & 2\left(x_{1}+x_{0}\right) /\left(x_{1}+x_{0}+1\right)-2\left(z_{1}+z_{0}\right) \\
d r_{1} / d t= & 0
\end{aligned}\right.
$$

It's obvious that the origin $O\left(x_{1}, y_{1}, z_{1}, r_{1}\right)=(0,0,0,0)$ is the equilibrium of system (3) when $r_{1}=0$, which has the same conclusion as the one $O\left(x_{0}, y_{0}, z_{0}, r_{0}\right)$ of system (2) through the analysis, stability and bifurcation types. The characteristic equation associated with system (3) is given by

$$
P(\xi)=\xi\left(\xi^{3}+Q_{1} \xi^{2}+Q_{2} \xi+Q_{3}\right)=0
$$

where $Q_{1}=-(A+E+I), Q_{2}=A E+A I+E I-B D-F H, Q_{3}=B D E+F H A-A E I$, and $A=-r_{0}-4 x_{0} /\left(x_{0}{ }^{2}+0.04\right)^{2}-\left(2956.581 x_{0}^{2} y_{0} z_{0}{ }^{4}\left(x_{0}-0.69\right)\right) /\left(\left(x_{0}+0.69\right)^{7}\left(z_{0}{ }^{4}+0.0016\right)\right)$, $B=\left(985.527 x_{0}^{3} z_{0}{ }^{4}\right) /\left(\left(x_{0}+0.69\right)^{6}\left(z_{0}{ }^{4}+0.0016\right)\right)+1$, $C=\left(6.3073728 x_{0}^{3} y_{0} z_{0}^{3}\right) /\left(\left(x_{0}+0.69\right)^{6}\left(z_{0}^{4}+0.0016\right)^{2}\right)$, $D=\left(4 x_{0}\right) /\left(x_{0}^{2}+0.04\right)^{2}+\left(2956.581 x_{0}^{2} y_{0} z_{0}^{4}\left(x_{0}-0.69\right)\right) /\left(\left(x_{0}+0.69\right)^{7}\left(z_{0}{ }^{4}+0.0016\right)\right)$, $E=-\left(985.527 x_{0}^{3} z_{0}{ }^{4}\right) /\left(\left(x_{0}+0.69\right)^{6}\left(z_{0}^{4}+0.0016\right)\right)-1$, $F=-\left(6.3073728 x_{0}^{3} y_{0} z_{0}{ }^{3}\right) /\left(\left(x_{0}+0.69\right)^{6}\left(z_{0}^{4}+0.0016\right)^{2}\right), G=2 /\left(x_{0}+1\right)^{2}, H=0, I=-2$.

When $r_{0}=7.49843$, characteristic roots of equilibrium $O=(0,0,0,0)$ of system (3) are $\xi_{1}=11.4501 i, \xi_{2}=-11.4501 i, \xi_{3}=-3.5845, \xi_{4}=0$ respectively.

$$
\begin{aligned}
& \text { Let }\left(\begin{array}{l}
x_{1} \\
y_{1} \\
z_{1} \\
r_{1}
\end{array}\right)=U\left(\begin{array}{l}
u \\
v \\
w \\
s
\end{array}\right) \text {, where } U=\left(\begin{array}{cccc}
0.6193 & -0.351 & -0.5359 & -0.05264 \\
0.6763 & 0 & 0.7658 & 0.15247 \\
-0.3988 & 0.041 & -0.0384 & -0.02685 \\
0 & 0 & 0 & 0.98654
\end{array}\right) \text {, system (3) becomes } \\
&\left.\qquad \begin{array}{l}
\text { \& } \\
\text { \& } \\
\text { \& } \\
\text { \& }
\end{array}\right)=\left(\begin{array}{cccc}
-3.5845 & 0 & 0 & 0 \\
0 & 0 & -11.4501 & 0 \\
0 & 11.4501 & 0 & 0 \\
0 & 0 & 0 & 0
\end{array}\right)\left(\begin{array}{l}
u \\
v \\
w \\
s
\end{array}\right)+\left(\begin{array}{l}
g_{1} \\
g_{2} \\
g_{3} \\
g_{4}
\end{array}\right)
\end{aligned}
$$

where

$$
\begin{aligned}
g_{1}= & 0.2658 u-0.2167 s+0.3456 v-0.275 w-3.1771 \sigma_{1}^{2} /\left(\sigma_{1}^{2}+0.04\right)-0.4923 \sigma_{1} \sigma_{3}, \\
& -4.2147 \sigma_{4} / \sigma_{5}-62.623 \sigma_{1}^{3} \sigma_{2} \sigma_{6} /\left(\left(\sigma_{2}+0.0016\right) \sigma_{7}^{6}\right)+1.0129 \\
g_{2}= & 1.0758 u-10.9085 s-0.8048 v+9.939 w+32.8752 \sigma_{1}^{2} /\left(\sigma_{1}^{2}+0.04\right)-4.3814 \sigma_{1} \sigma_{3}, \\
& -13.1192 \sigma_{4} / \sigma_{5}+647.9889 \sigma_{1}^{3} \sigma_{2} \sigma_{6} /\left(\left(\sigma_{2}+0.0016\right) \sigma_{7}^{6}\right)-6.4859 \\
g_{3}= & 2.0477 u-0.0078 s-11.7553 v-0.7571 w+68.097 \sigma_{1}^{2} /\left(\sigma_{1}^{2}+0.04\right)+0.4348 \sigma_{1} \sigma_{3}, \\
& +3.7221 \sigma_{4} / \sigma_{5}+1342.2289 \sigma_{1}^{3} \sigma_{2} \sigma_{6} /\left(\left(\sigma_{2}+0.0016\right) \sigma_{7}^{6}\right)-2.5616 \\
\sigma_{1}= & 0.0526 s-0.6193 u+0.351 v+0.5359 w-0.4, \\
\sigma_{2}= & (0.0268 s+0.3988 u-0.041 v+0.0384 w-0.2858)^{4}, \sigma_{3}=0.9865 s+7.4984, \\
\sigma_{4}= & 0.1053 s-1.2386 u+0.702 v+1.0718 w-0.8002, \\
\sigma_{5}= & 0.0526 s-0.6193 u+0.351 v+0.5359 w-1.4, \sigma_{6}=0.1525 s+0.6763 u+0.7658 w+1.2766,
\end{aligned}
$$




$$
\sigma_{7}=0.0526 s-0.6193 u+0.351 v+0.5359 w-1.09 \text {. }
$$

We can infer from the center manifold theory that there exists a center manifold of system (5), and its form can be expressed as follows:

$$
W_{l o c}^{c}\left(O_{1}\right)=\left\{(u, v, w, s) \in R^{4} \mid u=h^{*}(v, w, s), h^{*}(0,0,0)=0, D h^{*}(0,0,0)=0\right\}
$$

Let $h^{*}(v, w, s)=a_{1} v^{2}+b w^{2}+c s^{2}+d_{1} v w+e v s+f w s+\cdots$, the center manifold should meet

$$
D h^{*} \cdot\left(-11.4501 w+g_{2}, 11.4501 v+g_{3}, 0\right)^{T}+3.5845 h^{*}=g_{1}
$$

Now we have $a_{1}=-0.318, b=-0.3952, c=0.026, d_{1}=0.0571, e=0.0611, f=-0.0391$.

So the system confined by this center manifold of system (5) is

$$
\left(\begin{array}{c}
\& \\
\text { \& }
\end{array}\right)=\left(\begin{array}{cc}
0 & -11.4501 \\
11.4501 & 0
\end{array}\right)\left(\begin{array}{l}
v \\
w
\end{array}\right)+\left(\begin{array}{l}
f^{1}(v, w) \\
f^{2}(v, w)
\end{array}\right)
$$

where $f^{1}(v, w)=10.1919 v^{2}+31.7893 v w+13.9651 v^{3}+\cdots$, $f^{2}(v, w)=18.3368 v^{2}+57.3773 v w+25.1181 v^{3}+\cdots$.

Hence, it is not difficult to verify that:

$$
\begin{gathered}
a=\left.\frac{1}{16 \times 11.4501}\left(f_{v w}^{1}\left(f_{v v}^{1}+f_{w w}^{1}\right)-f_{v w}^{2}\left(f_{v v}^{2}+f_{w w}^{2}\right)-f_{v v}^{1} f_{v v}^{2}+f_{w w}^{1} f_{w w}^{2}\right)\right|_{(0,0)} \\
+\left.\frac{1}{16}\left(f_{v v v}^{1}+f_{v v w}^{2}+f_{w w w}^{2}\right)\right|_{(0,0)}=21.3992>0 \\
d=\left.\frac{d(\operatorname{Re}(\xi(s)))}{d s}\right|_{(0,0,0)}=8.404>0
\end{gathered}
$$

Summarizing the discussion above, we have the conclusion based on Hopf bifurcation theory.

Conclusion 1: A supercritical Hopf bifurcation occurs when $r$ passes through $r_{0}=7.4984261$ of system (2). When $r<r_{0}$, the equilibrium $O_{1}$ is stable. When $r>r_{0}$, the equilibrium $O_{1}$ will lose its stability, meanwhile the neighborhood around it will have an unstable periodic solution and system (2) begins to oscillate.

When $r_{0}=24.37676$, the system confined by the center manifold of system (3) is

$$
\left(\begin{array}{c}
\text { \& } \\
\text { \& }
\end{array}\right)=\left(\begin{array}{cc}
0 & 0.82473 \\
-0.82473 & 0
\end{array}\right)\left(\begin{array}{l}
v \\
w
\end{array}\right)+\left(\begin{array}{l}
f^{1}(v, w) \\
f^{2}(v, w)
\end{array}\right)
$$

where $f^{1}(v, w)=-1.6758 v^{2}-0.5822 v w+1.5245 v^{3}+\cdots$,

$$
f^{2}(v, w)=-0.7731 v^{2}-0.2605 v w+0.7239 v^{3}+\cdots \text {. }
$$

Conclusion 2 can be inferred when $a=0.3526>0$ and $d=-0.2817<0$.

Conclusion 2: A supercritical Hopf bifurcation occurs when $r$ passes through $r_{0}=24.37676$ of system (2). When $r<r_{0}$, the equilibrium $\mathrm{O}_{2}$ is unstable and system (2) is going to oscillate. However, when $r>r_{0}$, the equilibrium $\mathrm{O}_{2}$ is stable, and oscillatory phenomena of system (2) disappear.

\section{Numerical Simulation}

Fig. 1 is a bifurcation diagram of the equilibria of system (2). Each point of the curve (solid line) means the stable equilibrium, and the dashed line indicates unstable equilibria. Fig. 1 shows that the equilibrium $O(r)$ undergoes bifurcation two times, marked as HB1 and HB2 of which the corresponding parameter values are $r_{1}=7.4984261$ and $r_{2}=24.37676$ respectively. When $r<r_{1}$, there exists an stable equilibrium of system (2). When $r$ increases, the equilibrium loses its stability at $\mathrm{HB} 1$, and returns to be stable at HB2. 


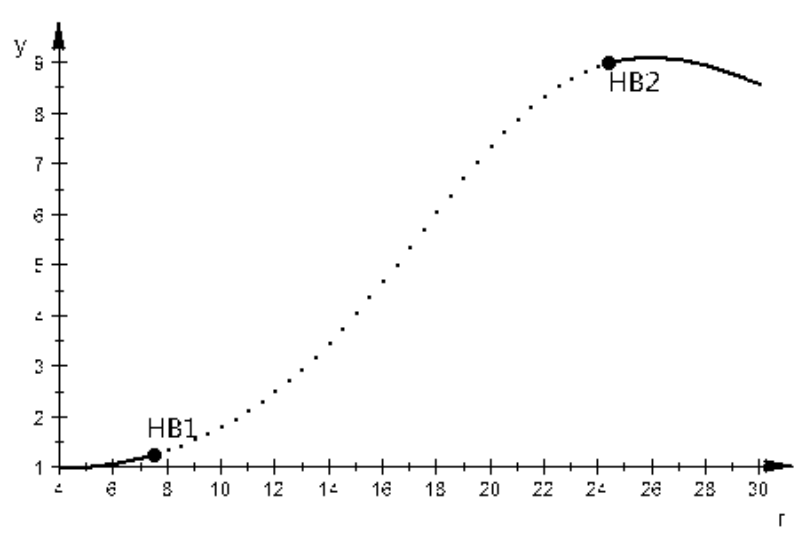

Fig. 1 Equilibria of system (2) in $(r, y)$-plane

Fig. 2(a) demonstrates a plot of orbit near a stable equilibrium of system (2), Fig. 2(b) shows time series of $x$ of system (2) for $r=7.49$. Numerical simulations illustrate that the equilibrium $O(r)$ is stable for $5<r<7.4984261$ and $24.37676<r<30$.

Fig. 3(a) indicates time series $t-x$ of system (2) for $r=24.3$ and Fig. 3(b) shows the stable periodic orbit of system (2) for $r=24.3$ and its period $T \approx 11.18$.
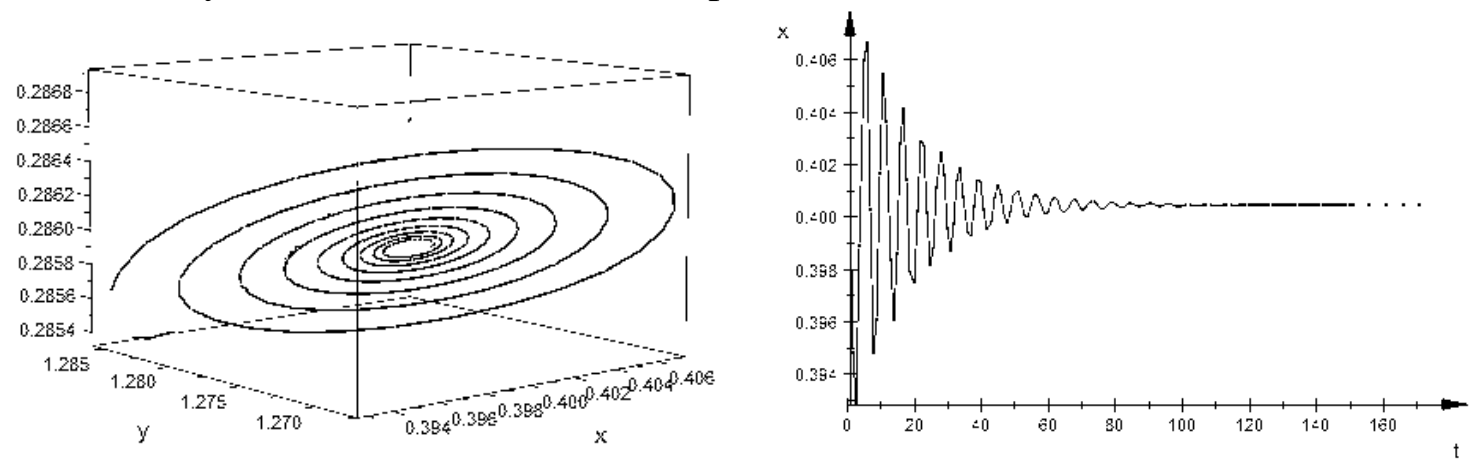

Fig. 2(a) Orbit near a stable equilibrium of system (2). Fig. 2(b) The time series $r=7.49$.

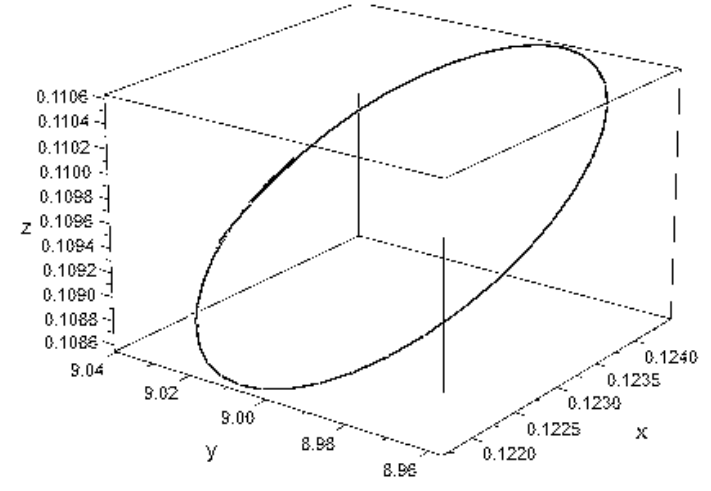

Fig. 3 (a) Stable periodic orbit.

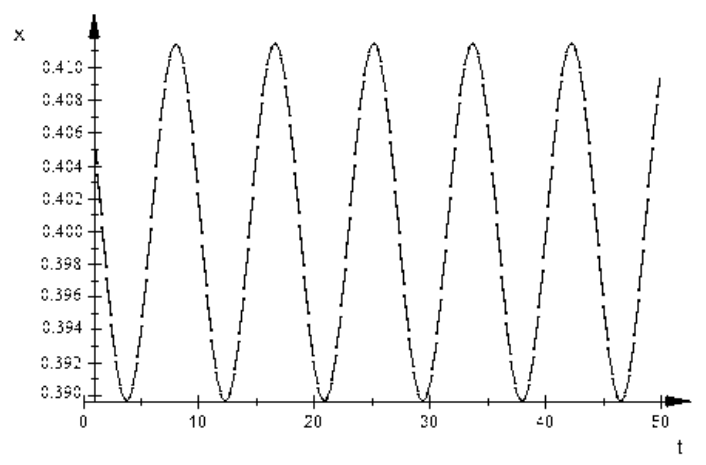

Fig. 3(b) The time series for $r=24.3$.

\section{Summary}

In this paper, we analyze the existence, types and stability of equilibria of Shen-Larter model of calcium oscillations by using the qualitative theory of dynamical systems and stability theory.

Based on the center manifold theory and bifurcation theory, we make a further study of the occurrence and disappearance of oscillations of this system, which proves that the phenomena are due to supercritical Hopf bifurcations of equilibria of this system.

Numerical simulations are carried out with the help of Matlab. The simulation results verify that they are effective and reasonable. 


\section{Acknowledgements}

The work in this paper has been supported by Key Project of University Excellent Youth Scholars of Anhui Province (No. gxyqZD2016300, gxyqZD2016303).

\section{References}

[1] J. Sneyd. “Models of calcium dynamics[J]”, Scholarpedia, vol.2, no.3, (2007), pp.1576-1582.

[2] M. J. Berridge, M. D. Bootman and P. Lipp. “Calcium - a life and death signal[J]”, Nature, vol.395, (1998), pp.645-648.

[3] M.J. Berridge, P.H. Cobbold and K.S. Cuthbertson. "Spatial and temporal aspects of cell signalling”, Philos. Trans. Roy. Soc. Lond. B Biol. Sci., vol.320, no.1199, (1998), pp.325-343.

[4] M.J. Berridge. "Inositol trisphosphate and calcium signaling”, Nature, vol.361, (1993), pp.315-325.

[5] Y. Tsunoda. “Oscillatory Ca2+ signaling and its cellular function”, New Biologist, vol.3, no.1, (1991), pp.3-17.

[6] R.W. Tsien and R.Y. Tsien. “Calcium channels, stores, and oscillations”, Ann. Rev. Cell Biol., vol.6, no.6, (1990), pp.715-760.

[7] K.S. Cuthbertson, and P.H. Cobbold. "Phorbol ester and sperm activate mouse oocytes by inducing sustained oscillations in cell Ca2+”, Nature, vol.316, (1985), pp.541-542.

[8] R. Jacob, J.E. Merritt, T.J. Hallam and T.J. Rink. "Repetitive spikes in cytoplasmic calcium evoked by histamine in human endothelial cells", Nature, vol.335, (1998), pp.40-45.

[9] R. Jacob. "Calcium oscillations in electrically non-excitable cells”, Biochem. Biophys. Acta., vol.1052, no.3, (1990), pp.427-438.

[10] M. Perc, M. Marhl. "Different types of bursting calcium oscillations in non-excitable cells", Chaos, Solitons \& Fractals, vol.18, no.4, (2003), pp.759-773.

[11] P. Shen, R. Larter. "Chaos in intracellular Ca2+ oscillations in a new model for non-excitable cells[J]”, Cell Calcium, vol.17, no.3, (1995), pp.225-232.

[12] Y. Chang, G. Chen. “Complex dynamics in Chen’s system[J]”, Chaos, Solitons \& Fractals, vol.27, no.1, (2006), pp.75-86.

[13] Z. Jing, C. Yu, G. Chen. "Complex dynamics in a permanent-magnet synchronous motor model[J]”, Chaos, Solitons \& Fractals, vol.22, no.4, (2004), pp.831-848.

[14] S Wiggins. Introduction to Applied Nonlinear Dynamical Systems and Chaos, Springer, Berlin (1990). 